\title{
Experience of starting ABO incompatible kidney transplantation in Bangladesh: report of seven cases
} \author{
Md. Sajid Hasan ${ }^{2}$, Abu Sayed ${ }^{3}$, Esrat Jahan Mitali ${ }^{4}$, Sheikh Anisul Haque ${ }^{5}$, Harun Ur Rashid ${ }^{1}$

\footnotetext{
${ }^{1}$ Department of Nephrology and Transplantation, Kidney Foundation Hospital and Research Institute, Dhaka, Bangladesh

${ }^{2}$ Department of Urology, Bangabandhu Sheikh Mujib Medical University, Dhaka, Bangladesh

${ }^{3}$ Department of Urology, Kidney Foundation Hospital and Research Institute, Dhaka, Bangladesh

${ }^{4}$ Department of Pathology, Kidney Foundation Hospital and Research Institute, Dhaka, Bangladesh

${ }^{5}$ Department of Transfusion Medicine, Impulse Hospital, Dhaka, Bangladesh
}

Nura Afza Salma Begum¹, Tasnuva Sarah Kashem¹, Mohammad Shakib Uz-Zaman Arefin ${ }^{1}$, AKM Khurshidul Alam²,

Background: $A B O$ incompatible kidney transplantations (ABOiKT) are increasingly being practiced worldwide. Currently, living kidney donation is the only viable option for transplantation in Bangladesh which is further restricted due to $A B O$ compatibility issue. We started ABOiKT in Bangladesh since 2018. Here we report our experience of seven ABOiKT cases.

Methods: The desensitization protocol included low-dose Rituximab (100 mg/body single dose), then plasma exchange. The replacement fluid used was fresh frozen plasma. Each plasma exchange was followed by intravenous immunoglobulin $5 \mathrm{gm} / \mathrm{dose}$. The immunosuppression consisted of Tacrolimus ( $0.1 \mathrm{mg} / \mathrm{kg} /$ day), Mycophenolate Mofetil $(1,500 \mathrm{mg} /$ day $)$ and Prednisolone $(0.5 \mathrm{mg} / \mathrm{kg} /$ day). All patients received basiliximab as induction therapy.

Results: Graft survival for our seven cases is $100 \%$ over a mean duration of 22 months. Mean creatinine was $204.6 \pm 47.4 \mu \mathrm{mol} / \mathrm{L}$. Two patients received intravenous methylprednisolone due to suspected acute cellular rejection. One patient required haemodialysis post-transplant due to delayed graft function and the graft function recovered subsequently. Two patients developed accelerated hypertension post transplantation. Two patients developed gastrointestinal disorders. One patient developed ST elevation myocardial infarction after plasma exchange which was managed conservatively and transplantation was performed 3 weeks later which was uneventful and post-transplant graft function was satisfactory. Infection was the major complication in our ABOiKT patients. Four out of seven patients $(57 \%)$ developed infection despite having prophylactic antibiotics and two patients $(28.6 \%)$ died with a functioning graft; one patient developed cytomegalovirus pneumonia and another patient developed pneumonia of unknown aetiology.

Conclusions: The start of ABOiKT in Bangladesh will substantially expand the living kidney donor pool and bring hope to a large number of end-stage renal disease patients who do not have $A B O$ compatible donor; hence reduce economic and health impact of long term dialysis therapy. However, Bangladesh is a high infection risk area and infection remains the major challenge in managing these patients. 